SCIENTIFIC REPORT

\title{
Transplantation of corneal endothelium with Descemet's membrane using a hyroxyethyl methacrylate polymer as a carrier
}

\author{
S Shimmura, H Miyashita, K Konomi, N Shinozaki, T Taguchi, H Kobayashi, J Shimazaki, \\ J Tanaka, K Tsubota
}

\begin{abstract}
Aims: To evaluate the histology and function of Descemet's membrane transplanted with intact endothelium.

Methods: Japanese white rabbits and human eyebank eyes were used as donors and recipients of Descemet's membrane transplantation. Donor endothelium was hydrodissected by injecting indocyanine green from a limbal incision, and then processed as a corneal scleral button. A $6 \mathrm{~mm}$ diameter donor sheet was trephined, and folded in half using a $6 \mathrm{~mm}$ diameter polymer as a carrier. Recipient endothelium was also hydrodissected from the limbus using trypan blue to stain the Descemet's membrane. Continuous curvilinear descemetorhexis (CCD) was performed to remove a circular section of the Descemet's membrane using a 27 gauge cystotome. Donor tissue was inserted into the anterior chamber through a $5 \mathrm{~mm}$ limbal incision and apposed to the host stroma. Polymers were removed following transplantation. Similar surgical procedures were performed in both rabbits and eyebank eyes. Haematoxylin eosin stains were performed after 28 days in rabbits, and eyebank eyes were fixed immediately following surgery for endothelial cell counts.

Results: Rabbit control eyes demonstrated stromal oedema caused by loss of Descemet's membrane, whereas transplanted eyes had clear corneas. The mean (standard deviation) pachymetry of operated eyes was 376.6 (SD 32.5) $\mu \mathrm{m}$ compared with 389.6 (SD 25.1) $\mu \mathrm{m}$ in the unoperated eye. Mean endothelial density immediately following surgery in eyebank eyes was 2749 (SD 288) cells $/ \mathrm{mm}^{2}$.

Conclusions: Transplantation of Descemet's membrane by $C C D$ produces a functional graft with an optically clear interface similar to control cornea.
\end{abstract}

$\mathrm{P}$ enetrating keratoplasty (PKP) has a long history as a surgical technique to treat irreversible opacification of the cornea. PKP is still the first choice of surgery for a wide range of diseases including bullous keratopathy, dystrophies, keratoconus, and trauma. However, damage spanning the entire thickness of the cornea from epithelium to endothelium is a relatively rare occurrence. For diseases other than bullous keratopathy, techniques such as deep lamellar keratoplasty (DLKP) ${ }^{12}$ or epithelial sheet transplantation $^{34}$ are new alternatives to PKP in which only damaged tissue is surgically replaced. Similarly, the stroma and epithelium are not necessary in the treatment of bullous keratopathy, where only the endothelium is compromised by disease or injury. Several studies have shown the feasibility of transplanting posterior lamellar tissue instead of PKP.
One such technique is endothelial lamellar keratoplasty which uses a microkeratome to create a flap, followed by the replacement of posterior lamellar tissue taken from a donor cornea. ${ }^{56}$ The use of a microkeratome leaves little opacification of the stromal interface, and a large flap covering the donor offers greater adhesive strength than running sutures used in PKP. Another method for transplanting endothelial tissue with a thin layer of stroma used as a carrier was reported by Melles et al. ${ }^{7}$ The technique was further refined to allow transplantation through a small limbal incision-as opposed to a large wound reported in the original technique. ${ }^{8}$ Initial clinical results are promising; however, manual lamellar incision of the stroma may cause mild haze in the stromal interface.

The adhesion between Descemet's membrane (DM) and posterior stroma is not anatomically strong, so the membrane can be peeled off without much effort. This is done when removing donor endothelium from a DLKP donor button before transplantation. ${ }^{9}$ DM can also be detached by hydrodissection or viscodissection to obtain a smooth interface at the plane separating DM and stroma. ${ }^{10}$ Our experience with DLKP by hydrodissection led to a new technique to obtain controlled sizes of DM by hydrodissecion followed by continuous curvilinear descemetorhexis (CCD). However, Descemet's membrane alone has the tendency to curl into a tube-like structure with the endothelial cells facing outwards. In order to control the plasticity of DM, we used polymers as a carrier during insertion and spreading of DM. In the present study, we show the histopathological and functional results of endothelial transplantation in rabbit and human eye bank eyes by this technique.

\section{METHODS}

Japanese white rabbits (female, $3 \mathrm{~kg}$ body weight, Shiraishi experimental animal breeding farm, Tokyo, Japan) were used as an animal model for endothelial transplantation. All animals were treated according to the ARVO Statement for the Use of Animals in Ophthalmology and Vision Research. Recipient animals $(\mathrm{n}=4)$ were anaesthetised with $4 \mathrm{ml}$ intramuscular ketamine and xylazine (1:7 mixture) and topical xylocain. To limit the extensive fibrin response observed in rabbits, pupils were expanded with topical tropicamide, and the anterior chamber was irrigated with heparin $(5 \mathrm{U} / \mathrm{ml})$. A $5 \mathrm{~mm}$ incision was made at the corneoscleral rim down to the plane of Schlemm's canal, and DM was hydrodissected by injecting $0.04 \%$ trypan blue into the supra Descemet space using a blunt 27 gauge needle (fig $1 \mathrm{~A}-\mathrm{C}$ ). Following injection of air into the anterior

Abbreviations: $C C D$, continuous curvilinear descemetorhexis; DLKP, deep lamellar keratoplasty; DM, Descemet's membrane; HE, haematoxylin eosin; PKP, penetrating keratoplasty. 

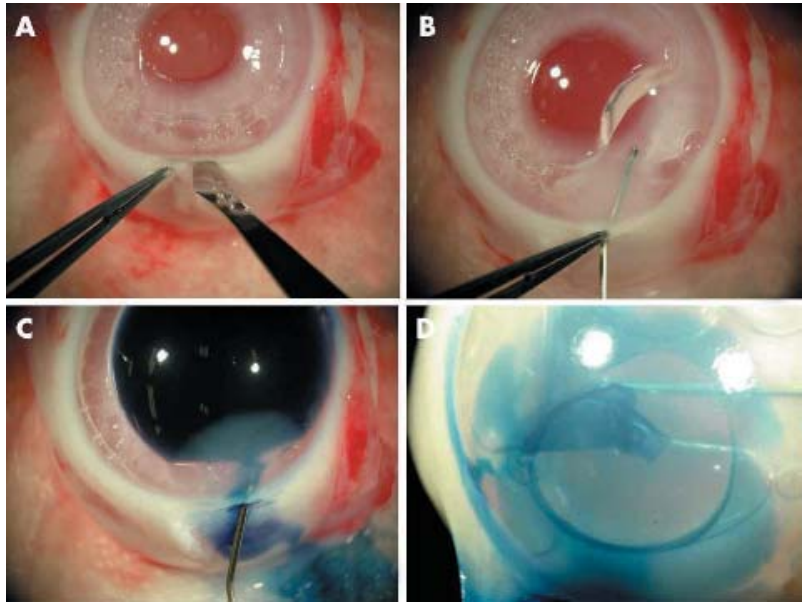

Figure 1 Surgical procedure for preparing the recipient shown in a rabbit eye. (A) An incision was made down to the plane of DM.

(B), (C) Hydrodissection of DM is performed with balanced salt solution followed by trypan blue. (D) After removing trypan blue dye, a cystotome is inserted into the anterior chamber to perform CCD. The same procedure was performed in eyebank eyes.

chamber, CCD was performed to remove a circular section of the Descemet's membrane using a 27 gauge cystotome (fig 1D).

Two animals were sacrificed using an overdose of pentobarbital at the time of surgery to provide a total of four donor buttons. Following enucleation of the donor globe, a $3 \mathrm{~mm}$ incision was made at the corneoscleral rim, and DM was hydrodissected by injecting $6.25 \mathrm{mg} / \mathrm{ml}$ indocyanine green so that the donor and recipient DM could be differentiated by colour. After creating a corneal scleral button, a $6 \mathrm{~mm}$ diameter incision was made in the DM using a manual trephine, and the endothelium was coated with viscoelastic material to protect the cells from physical friction. The polymer/DM composite was folded in half using an $8 \mathrm{~mm}$ diameter hyroxyethyl methacrylate polymer (one day ACUVUE; Johnson and Johnson, Jacksonville, FL, USA) as a carrier (fig 2A). The polymer/DM with endothelial cells facing inward was grasped with capsulorrhexis forceps towards the leading edge of the polymer (fig 2B, C). Donor tissue with the polymer carrier was inserted into the anterior chamber filled with air through the $5 \mathrm{~mm}$ limbal incision, and apposed to the host stroma by expanding the polymer. Additional air is injected into the anterior chamber to further adhere the DM to the posterior stromal surface. Polymers were removed at the end of surgery. Control animals $(n=4)$ were operated by CCD alone without transplantation of donor endothelium, and another four rabbits served as sham negative control. All animals received topical antibiotics (levofloxacin) and steroids (betamethasone). After observing the rabbits for 28 days, animals were sacrificed and recipient corneas were fixed with $10 \%$ formalin over night. Paraffin sections were stained with haematoxylin and eosin.

Human eye bank eyes (five donors globes, five recipient globes) were obtained from the Northwest Lions Eyebank (Seattle, WA, USA). Donor DM were transplanted into five globes according to the procedure described above. Transplanted eyes were immediately processed into corneal scleral buttons for histological analysis of the transplanted endothelium. Following fixation with $4 \%$ paraformaldehyde, endothelial cell density was calculated by staining the nuclei of cells with 4', 6-diamidino-2-phenylindole dihydrochloride (DAPI, Dojindo Laboratories, Kumamoto, Japan) and analysed using the NIH Image software (National Institute of Health, Bethesda, MA, USA).
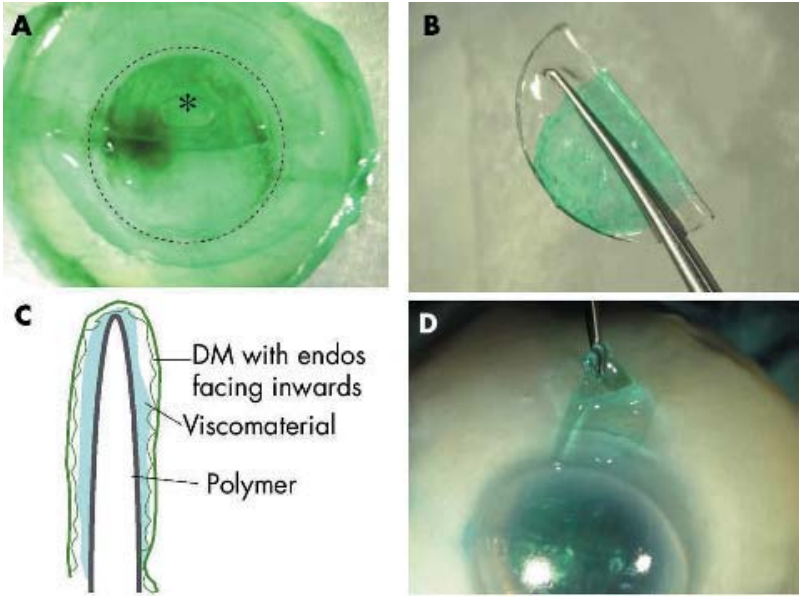

Figure 2 Preparation of donor tissue shown in an eyebank eye. (A) Donor DM is dissected with indocyanine green as described in figure 1, processed into a corneoscleral button, and trephined so that an incision is made in DM (dotted circle). Viscomaterial is coated on the endothelial surface and a hyroxyethyl methacrylate polymer carrier $\left(^{*}\right)$ is folded in half and placed on DM. (B) Capsulorrhexis forceps used to grasp the polymer/DM composite with endothelial cells facing inwards. (C) A cross sectional schematic view of polymer/DM shown in (B).

(D) The polymer is removed after DM is placed in position with the use of air. The same procedure was performed in rabbits.

\section{RESULTS}

\section{Endothelial transplantation in rabbits}

The left eyes of four rabbits were transplanted with donor DM. Figure 3A shows a transplanted eye following surgery; the transplanted DM is stained green and the host DM is stained blue. Figure 3B shows the transplanted eye with a clear central cornea 28 days following surgery. Control eyes (fig 3C) had extensive stromal oedema with minimal view of the anterior chamber. The mean (standard deviation) pachymetry reading of transplanted eyes was 407.2 (SD 63.0) $\mu \mathrm{m}$, which was not significantly different compared with sham operated controls with an average thickness of 391.2 (SD 20.8) $\mu \mathrm{m}$. Positive control eyes operated by CCD alone were too oedematous to obtain readings with the pachymeter used in our study. The mean cell density in the rabbit eye 28 days after surgery was 2201.3 (SD 441.5) cells/
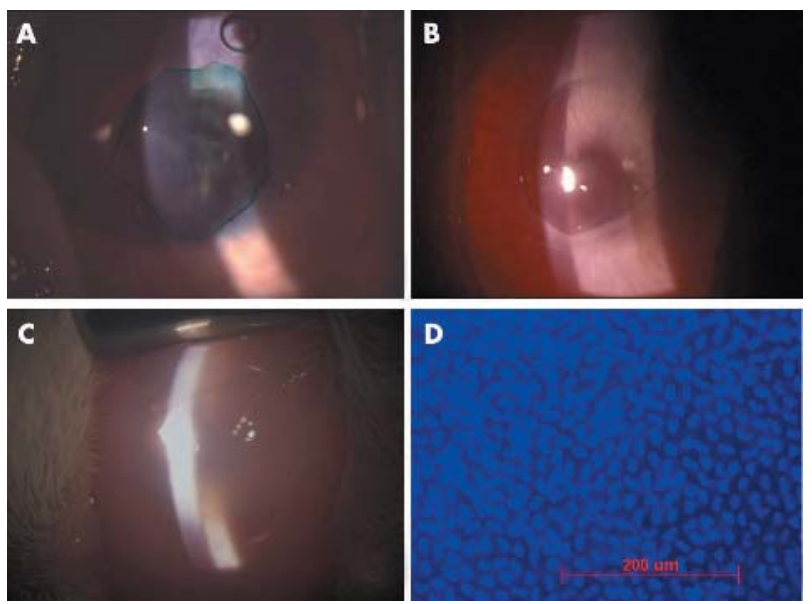

Figure 3 Postoperative eye in a rabbit with DM transplantation immediately following surgery (A) and after 28 days (B). Control eyes stripped of DM had extensive stromal oedema and opacification (C). Histology of endothelium within the graft shows a uniform layer of endothelium (D). 


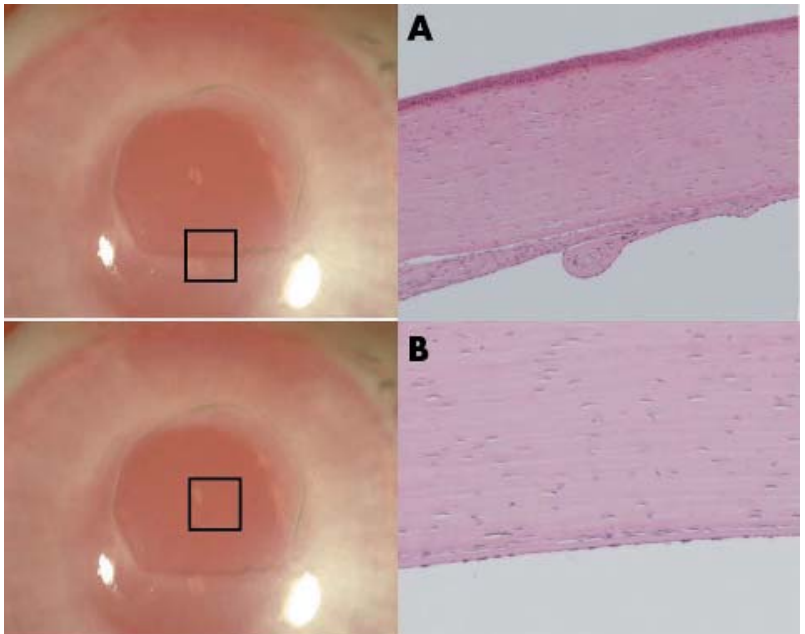

Figure $4 \mathrm{HE}$ stains of the edge (A) and centre (B) of donor show a well apposed DM with slight curling of the edge. Original magnification $(\times 100)$ for HE stains.

$\mathrm{mm}^{2}$ (fig 3D) compared with 3180.5 (SD 98.2) cells $/ \mathrm{mm}^{2}$ in sham control. Cell loss was greater in rabbit eyes compared with eye bank eyes because of the very shallow anterior chambers in rabbits, which was an obstacle during surgical procedures.

Animals were sacrificed after 28 days, and the transplanted eyes were processed for histological examination. Haematoxylin eosin (HE) stains of the peripheral graft show a slightly curled edge of the transplanted DM (fig. 4A). The central graft is attached to the host stroma (fig 4B), which under high magnifications shows how the transplanted DM resembles DM in sham operated controls (fig 5). There was no observable infiltration of inflammatory cells in any of the operated eyes after 28 days.

\section{Endothelial transplantation in human eyebank eyes}

A total of five "donors" were transplanted into another five eyebank eyes using the same procedure explained in detail above. Corneal scleral rims were processed immediately following implantation to show the positioning of the graft (fig 6). Nuclear stains using DAPI revealed an endothelial cell density of 2749 (SD 288) cells $/ \mathrm{mm}^{2}$, suggesting that an adequate density of endothelial cells can be transplanted using this technique. No information was available as to the
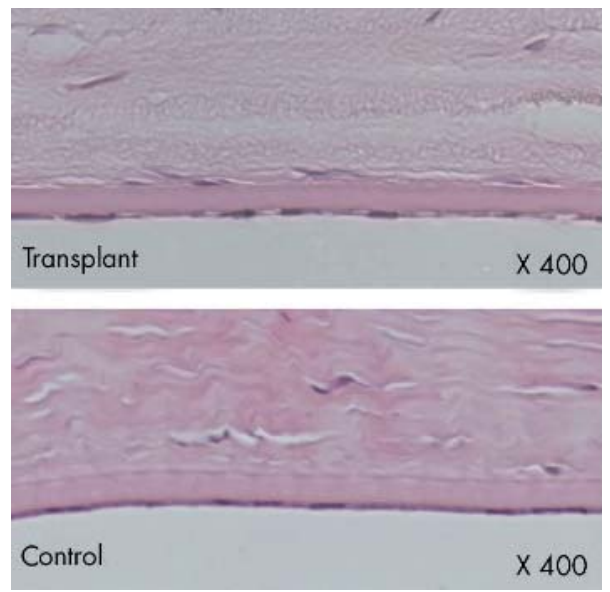

Figure 5 Higher magnification $(\times 400)$ of the central graft reveals similar histological findings in both the transplanted eye and control.

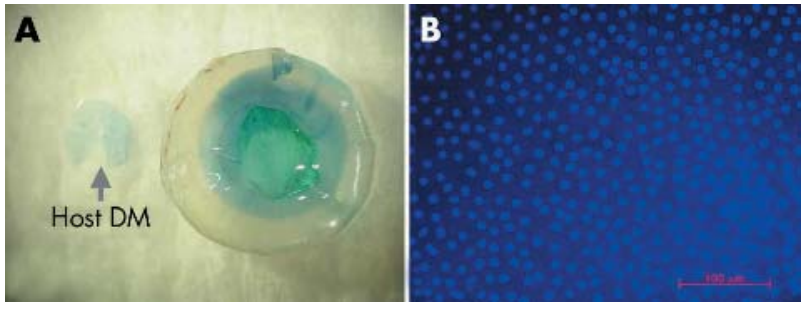

Figure 6 DM transplantation in human eyebank eye with green donor DM and blue recipient DM following CCD (A). Average endothelial density immediately following insertion of donor DM was 2749 (SD 288) cells $/ \mathrm{mm}^{2}$.

cell density of the eyes before the experiment; therefore the endothelial cell loss rate was not calculated.

\section{DISCUSSION}

Surgical techniques in keratoplasty have changed over the past few years towards reducing tissue damage to a minimum, while transplanting only the layers of the cornea that are necessary for the particular patient. For stromal diseases such as keratoconus and the various dystrophies, DLKP can remove all of the stroma while leaving the host endothelium intact. ${ }^{12}$ Epithelial transplants using amniotic membrane carriers are already in clinical use for the treatment of acute phase burns and Stevens Johnson syndrome with persistent epithelial defects. ${ }^{11}$ The same technique can also be used to restore vision in the chronic stage of cicatricial disease such as ocular cicatricial pemphigoid, if the indications are chosen carefully.

Bullous keratopathy, however, is still mainly treated by PKP as the techniques involved in transplanting endothelium were believed to be technically difficult. Several experimental reports using animals have attempted to transplant endothelium by seeding the cells on hydrogel polymers, ${ }^{12}$ or by using bovine DM as carriers. ${ }^{13}$ More recently, clinical studies have described techniques to transplant the posterior stroma, including the endothelium, into bullous keratopathy patients. One technique is reported by Azar et al, who used a microkeratome to create a flap so that the posterior half of the donor cornea could be transplanted beneath the flap. ${ }^{6}$ Our experience with the technique is favourable, with a clear optical interface between the host and donor. However, epithelial ingrowth can be a complication, and the need of a keratome can be costly. Another method described by Melles et al uses a spatula to dissect the host Descemet's membrane, after which a donor DM is inserted into the anterior chamber though a small corneal scleral incision. ${ }^{78}$ The procedure seems promising in terms of refraction; however, stromal haze in the donor-host interface may occur when a spatula or knife is used to create a lamellar incision in the deep stroma. A custom made scraper was recently reported by the same group, which may solve these issues in the repicient stromal bed. ${ }^{14}$

The method described in this study uses hydrodissection to dissociate Descemet's membrane in both the donor and host, without the use of special instruments. This creates clear cut dissociation at the interface of DM and stroma, because fluid enters into the plane of weakest adhesion. The interface in this case is smoother than other methods using blades or spatula and, furthermore, does not interrupt the posterior stroma that contains keratocytes which may become activated to cause tissue reaction. The high magnification in figure 5 shows how the transplanted DM is closely apposed to the host stroma with little cellular infiltration or scarring in the posterior stroma. The grafts in rabbits show no interface 
opacity under slit lamp examination, and the endothelial cell count is within an acceptable range. The average cell density in rabbits was lower than human eyebank eyes in our study, which was due to the fact that surgical manipulation was much more difficult in rabbits, which have very shallow anterior chambers and show fibrinotic response during surgery.

The rabbit data show that transplanted DM is functional, and that little tissue reaction is observed at the host-donor junction. However, as rabbit endothelial cells are known to proliferate in vivo, the same experiment was done using human eyebank eyes. The objective was to simulate a clinical situation, and also to assess the loss of endothelial cells during the procedure. As shown in figure 6, controlling the size and apposition of donor DM is possible, and the average endothelial cell density was above 2500 cells $/ \mathrm{mm}^{2}$. The preoperative endothelial density was not available; however, considering that the eyes were preserved in moist chambers without the use of storage medium, the results can be said to be more than adequate.

The largest obstacle to manipulating DM is the tendency for the $10 \mu \mathrm{m}$ thick membrane to curl up with the endothelium facing outwards. Melles et al have developed an injector for the purpose of inserting the membrane into the anterior chamber. ${ }^{10}$ We have shown that a hydroxyethyl methacrylate polymer can be used as a carrier by folding the DM in half, and also protect the graft from the loss of endothelial cells during insertion into the anterior chamber. The polymer can then be unfolded in the anterior chamber, and also be used to appose the DM to the stroma. The inserted DM is unfolded using a spatula inserted into the plane opposite the endothelium. Although the donor edge is not necessarily a perfect fit with the recipient bed (fig 4), the adhesion of donor DM is firm as evidenced from the fact that the membrane remains attached following paraffin fixation during HE stains. The report by Melles et al also shows that a perfect match is not necessary.

Transplantation of hydrodissected DM is an effective means to reproduce the normal anatomy of the posterior stroma. The hydrostatic pressure exerted by the endothelial pump is sufficient for the immediate attachment of the donor $\mathrm{DM}$, and a polymer carrier offers protection and a means to insert DM through a small surgical wound. Encouraged by the results of this study, we are currently preparing a clinical trial based on the surgical technique described here.
Authors' affiliations

S Shimmura, H Miyashita, K Konomi, N Shinozaki, J Shimazaki, K Tsubota, Department of Ophthalmology, Tokyo Dental College, Chiba, Japan

T Taguchi, H Kobayashi, J Tanaka, Biomaterials Center, National Institute for Materials Science, Ibaragi, Japan

K Tsubota, Department of Ophthalmology, Keio University, Tokyo, Japan

This study was supported by The Advanced and Innovational Research Program in Life Sciences from the Japanese Ministry of Education, Culture, Sports, Science and Technology.

Correspondence to: Dr S Shimmura, Department of Opthalmology, Toyko Dental College, 5-11-13 Sugano, Ichikiawa, Chiba 272-8513, Japan; shimmura@tdc.ac.jp

Accepted for publication 7 July 2004

\section{REFERENCES}

1 Aggarwal RK. Deep lamellar keratoplasty-an alternative to penetrating keratoplasty. Br J Ophthalmol 1997;81:178-9.

2 Sugita J, Kondo J. Deep lamellar keratoplasty with complete removal of pathological stroma for vision improvement. $\mathrm{Br} J$ Ophthalmol 1997;81:184-8.

3 Tsai RJ, Li LM, Chen JK. Reconstruction of damaged corneas by transplantation of autologous limbal epithelial cells. N Engl J Med 2000:343:86-93.

4 Rama P, Bonini S, Lambiase A, et al. Autologous fibrin-cultured limbal stem cells permanently restore the corneal surface of patients with total limbal stem cell deficiency. Transplantation 2001;72:1478-85

5 Busin M, Arffa RC, Sebastiani A. Endokeratoplasty as an alternative to penetrating keratoplasty for the surgical treatment of diseased endothelium: initial results. Ophthalmology 2000;107:2077-82.

6 Azar DT, Jain S, Sambursky R, et al. Microkeratome-assisted posterior keratoplasty. J Cataract Refract Surg 2001;27:353-6.

7 Melles GR. Posterior lamellar keratoplasty. Arch Soc Esp Oftalmol 2002;77:175-6.

8 Melles GR, Lander F, Nieuwendaal C. Sutureless, posterior lamellar keratoplasty: a case report of a modified technique. Cornea 2002;21:325-7.

9 Shimmura S, Shimazaki J, Tsubota K. Therapeutic deep lamellar keratoplasty for cornea perforation. Am J Ophthalmol 2003;135:896-7.

10 Melles GR, Lander F, Rietveld FJ. Transplantation of Descemet's membrane carrying viable endothelium through a small scleral incision. Cornea 2002;21:415-18

11 Koizumi N, Inatomi T, Suzuki T, et al. Cultivated corneal epithelial transplantation for ocular surface reconstruction in acute phase of StevensJohnson syndrome. Arch Ophthalmol 2001;119:298-300.

12 Mohay J, Lange TM, Soltau JB, et al. Transplantation of corneal endothelial cells using a cell carrier device. Cornea 1994;13:173-82.

13 Lange TM, Wood TO, McLaughlin BJ. Corneal endothelial cell transplantation using Descemet's membrane as a carrier. J Cataract Refract Surg 1993; 19:232-5.

14 Melles GR, Wijdh RH, Nieuwendaal CP. A technique to excise the descemet membrane from a recipient cornea (descemetorhexis). Cornea 2004;23:286-8. 